\title{
The Sites of the Latin American Giant Observatory
}

\author{
Iván Sidelnik ${ }^{* \dagger}$ \\ Laboratorio de Neutrones y Reactores, Centro Atómico Bariloche and Instituto \\ Balseiro-UNCuyo \\ and Consejo Nacional de Investigaciones Científicas y Técnicas, \\ Av. Bustillo 9500, R8402AGP, S.C. de Bariloche, Río Negro, Argentina \\ E-mail: sidelnikecnea.gov.ar \\ for the LAGO Collaboration \\ see the full list of members and institutions at http://lagoproject.org/colab.html \\ E-mail: lago-pi@lagoproject.org
}

The Latin American Giant Observatory (LAGO) is an extended cosmic ray observatory, which consists on a wide network of water Cherenkov detectors located in nine different countries. The geographic distribution of the LAGO sites, with different altitudes and geomagnetic rigidity cut offs, combined with the new electronic system for control, atmospheric sensing and data acquisition on board of each detector, allow the realization of multiple and variated astrophysics, space physics and atmospheric physics studies at regional scale. In this work it will be described the LAGO sites and the capabilities of the LAGO detection network spanned across Latin America.

The 34th International Cosmic Ray Conference,

30 July- 6 August, 2015

The Hague, The Netherlands

\footnotetext{
* Speaker.

${ }^{\dagger}$ Presenter: Alberto Carramiñana
} 


\section{Introduction}

LAGO (Latin American Giant Observatory) is a collaboration that comes from the association of Latin American astroparticle researchers [1]. It started in 2006 and it was originally designed to survey the high-energy component of Gamma Ray Bursts (GRBs) [2]. It is a network of groundbased water Cherenkov detectors (WCDs), located at different altitudes, between sea level and mount Chacaltaya at more than $5200 \mathrm{~m}$ a.s.l., with also a large range of geomagnetic rigidity cutoffs (RC). This collaboration was motivated by the experience of the Pierre Auger Observatory, and one of its primary objectives is to install WCDs along different sites in Latin America. At the moment LAGO is composed by ten countries forming a non-centralized and collaborative network of 25 institutions. The countries are: Argentina, Bolivia, Brazil, Colombia, Ecuador, Guatemala, México, Perú and Venezuela and the recent incorporation of institutions from Spain. 86 people integrate the LAGO community. It results very important to notice that this is a collaboration to search for space phenomena and understand its physics, working also as an integration network of science. LAGO operates as a connection for a lot of universities developing educational project to help physics students to deal with the construction of detectors, data acquisition and analysis, along to its physics interpretation.

Although the original plan comprised the detection of the high energy component of GRBs, recently it has been shown that WCDs can also be used to study the Solar Modulation (SM) of Galactic Cosmic Rays (GCR) and other transient phenomena, by measuring the variations of the flux of secondary particles at ground level [3]. Measuring the flux modulation of the GCR at different locations on Earth that span over a wide range of rigidity cut offs and using the same type of detectors, can provide important information about the global structure of the magnetic cloud reaching the space environment surrounding the Earth. Astroparticle studies in the context of GRBs [4, 5, 6], space weather and background radiation at ground level [7] are the main scientific objectives of the Latin American Giant Observatory [1].

This works describes the new detector concept as an instrument that measures the physical quantities that are necessary to perform the analysis of low energy astroparticles at ground level. This is shown in section 2. In section 3 there is an overview of the different characteristics of the sites of LAGO in the Latin American region. Finally in section 4 we give some final remarks.

\section{The detector}

The WCDs of LAGO are composed by a water container with a reflective material inside, and a photomultiplier tube (PMT) to convert the Cherenkov radiation produced by the interaction of the high energy particles with water in to an electric signal. The response of each WCD, like other particle detectors, are short electric pulses ( $\sim 200 \mathrm{~ns}$ ) that are recorded by high speed (240 MHz) data acquisition systems. A typical WCD of one of the LAGO sites is shown in the right side of Figure 1.

This respond to the concept of a detector working as an isolated station that transmit data to a centralized place. The new idea that is showed in section 2.1 develop a concept of a multi use station. 

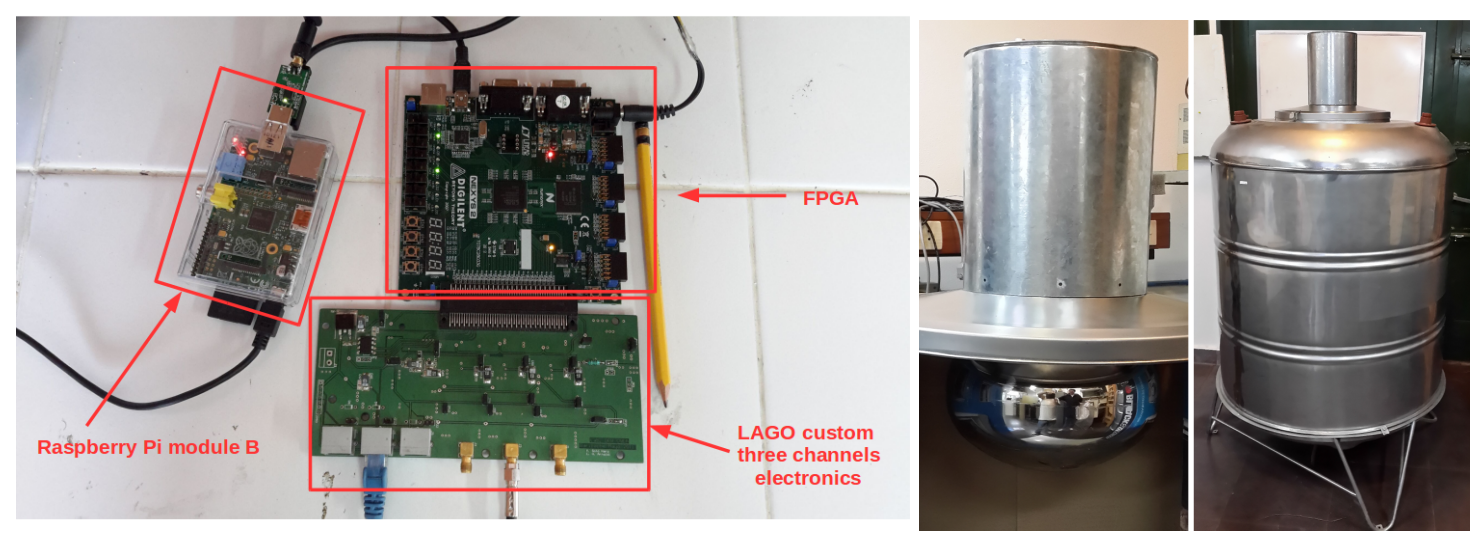

Figure 1: LAGO electronics: It can be seen the raspberry pi model B, the board with the Nexys2 FPGA and the custom three channels digitazer board.

Regarding the WCD, the PMT is connected to a electronic board that have a voltage divider, a high voltage supply, and an amplifier of the output pulse. The high voltage supply can be controlled typically from $0 \mathrm{~V}$ to $2000 \mathrm{~V}$ with a signal from $0 \mathrm{~V}$ to $2.5 \mathrm{~V}$. The output pulse shape of the PMT depends on the geometry of the detector, for a tank of 10001 the FWHM ${ }^{1}$ of the pulse is around $150 \mathrm{~ns}$. The data acquisition and high voltage parameters of the experiment can be settled by a PC program and the acquire pulses are transfered in real time to the PC or SBC via a USB interface. This system has the capability of acquire three or four independent channels and provide the control signal for the three or four HV supply.

The data acquisition system includes a custom electronic board that integrate the front-end electronics: three ADC, anti-aliasing filters, baseline control control circuit, high voltage control signal, and the necessary service circuits. This board is connected to a NEXYS-2 board via a high speed FX2-Hirose connector. The NEXYS2 have a FPGA Spartan3E that is connected to the PC with a USB interface. The USB interface between the PC and the FPGA is managed with a Cypress chip included in the board. In the FPGA there is an implementation of a module of command, that provided connectivity with the sensors the GPS and with a pre-processor that filter the input pulses. The pulses that have an amplitude over a trigger level are transferred to the PC.

Recent developments of Single Board Computers (SBC) as the Raspberry Pi and arduino [8, 9], allow us to made an integral and autonomous station, with low power consumption that has the capability of perform analysis, encoding, and data transfer. The Raspberry Pi board that controls the station is a model B, showed in Figure 1, left. Also, it is shown the whole LAGO electronic that can be seen with the different components. The NEXYS-2 FPGA board is showed with the digitalized board custom made with three or four available channels, depending the number of detectors available.

Since the LAGO data analysis needs to take into account the influence of atmospheric effects, such as pressure or air temperature, each LAGO WCD is equipped with several environmental sensors. These measurements represent an opportunity to provide environmental information to other communities, like for example, ecologists studying the high altitude environments to correlate

\footnotetext{
${ }^{1}$ Full Width at Half Maximum
} 
for possible climate change and global warming effects.

\subsection{New detector concept}

The need to measure different groups of physical magnitudes in remote and difficult to access places, or with almost no infrastructure, requires the implementation of autonomous and reliable devices. This kind of apparatus might be able to handle the access to different peripheral sensors that should be controlled independently and may need to manage different time stamps for the incoming data.

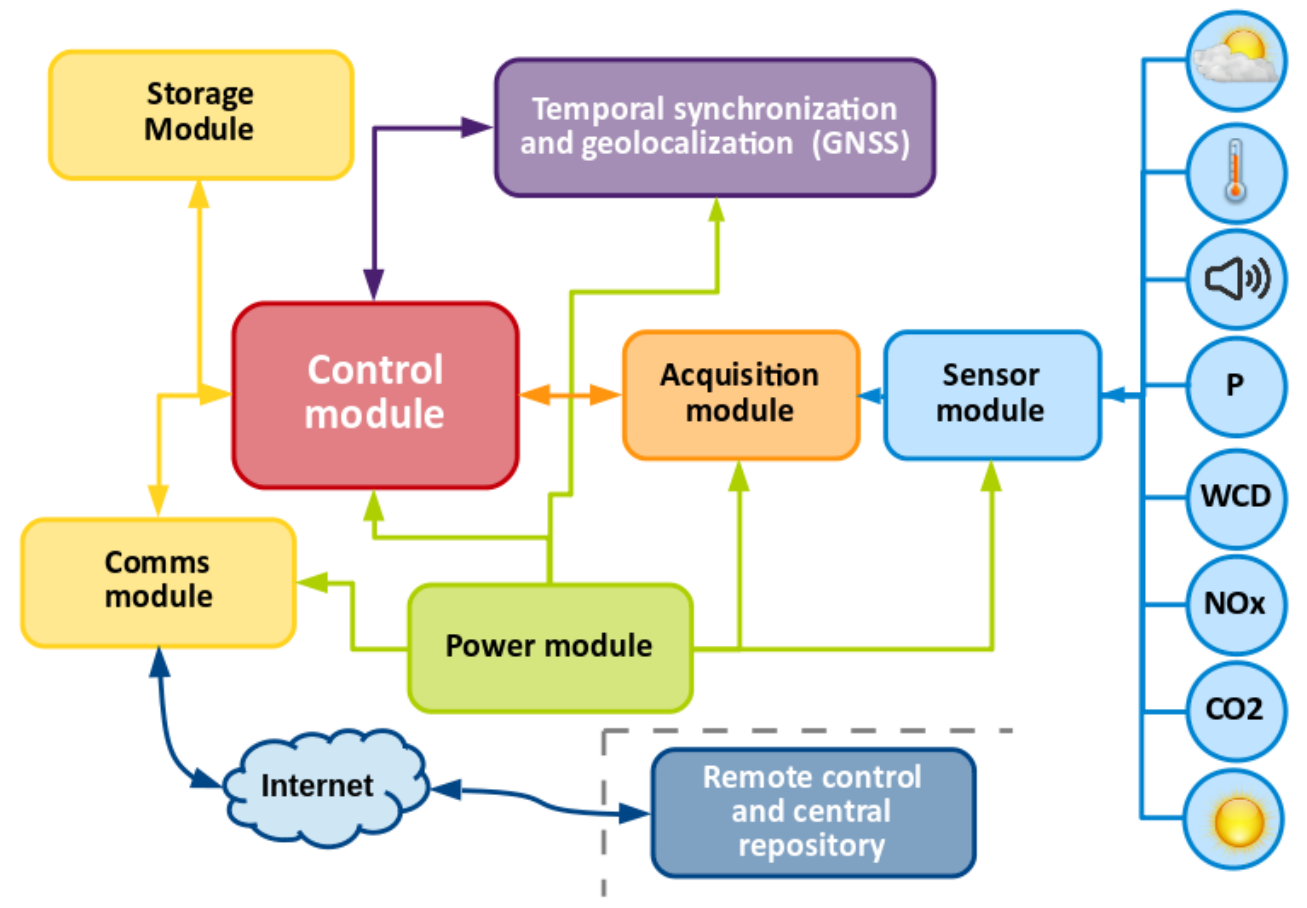

Figure 2: Block diagram of the autonomous station for the new LAGO detector concept. The constituents of the station are highlighted: the data acquired by the different sensors that are in the peripheral are sent to the sensor module and from there to the acquisition module for its digitalization or channeling trough the $\mathrm{I}^{2} \mathrm{C}$ bus. The digitalized data are resented to the control module for temporal identification, storage and transmission to a remote repository.

In Figure 2 it is shown a diagram of the autonomous and remote measurement station. This new concept for the LAGO detector shows how the instrument that measures the particles (the WCD) can be treated as an peripheral element. This autonomous measurement is built from the integration of different independent modules that adds different functionalities to the station.

The modules that composes a LAGO station are listed below:

Sensor module: built to support the different sensors. These sensors are integrated in to a board Arduino-shield [10] of customary design. We use commercial, low cost and low consumption sensors. 
Acquisition and digitization module: data acquisition and digitization is performed in a board based in the Arduino are microcontroller ATmega328 [11]. The data bus allows the reading and digitization of up to six analogous sensors, fourteen digital sensors and a communication bus based on the $\mathrm{I}^{2} \mathrm{C}$ standard.

Control, encoding, storage and transport module: This module is supported by and SBC Raspberry Pi B/B+ and recently Pi 2 [12]. All station operations are controlled within this module. The digitized data by the acquisition module are conformed, classified and stored following a priori established parameters. The flexibility that gives the operative system of the SBC, GNU/Linux based, gives the chance to perform some on-line local analysis of the data. In case of need, it also gives the possibility of remotely access, through a communication module. Data is storage using SSD solid state devices of $1 \mathrm{~TB}$, that guarantees low power consumption and with that the capability of operation at remote places.

Power supply module: Low power consumption has been a mandatory design parameter to take in to account for the assemble of an autonomous station. With a maximum consumption of $10 \mathrm{~W}$ this detector can be operate in remote places using solar panels and batteries.

Geolocalization module: This module allows to incorporate Global Navigation Satellite System (GNSS) devices compatible with the NAVSTAR-GPS/GLONASS/GALILEO system. This gives the chance to determine the position of the station and temporal synchronization of the signal with a resolution of $\lesssim 20 \mathrm{~ns}$ [13]. The selected devices will allow also to perform total electron content (TEC) analysis [14].

Communication module: The design of this module depends on the communication network available at the place of operation of the detector. Currently there are different sub modules available that can stablish links at different velocities through the standards: IEEE 802.3 (ethernet), IEEE 802.11 (WiFi), IEEE 802.16 (WiMax), IEEE 802.15.1 (Bluetooth), GPRS (2.5G and $3 \mathrm{G}), 4 \mathrm{G}-\mathrm{LTE}$.

\section{The sites of the Latin American Giant Observatory}

LAGO spans over many different countries in Latin America. In this section we describe the sites according to the location in each of the countries that have or will have a LAGO detector. In Fig.3, left, we show a map of the Latin American region from Mexico to the Antarctic. In this map the blue triangles shows the sites that have at least one operational detector, and in red squares those who are to be installed in the 2015-2016 period.

The plot in Figure 3, right, shows the rigidity cut offs, in GV, for the latitudes connected with the map on the left $[7,15]$. It can be appreciated the spanning around similar geographical longitudes but different values of rigidities that can be characterized by the LAGO network. Then, by combining all the data measured at several locations of our detection network and analyzed by using the multi-spectral technique[7], the LAGO project will provide detailed and simultaneous information at different energy ranges of the disturbances produced by transients and long term space weather phenomena. 

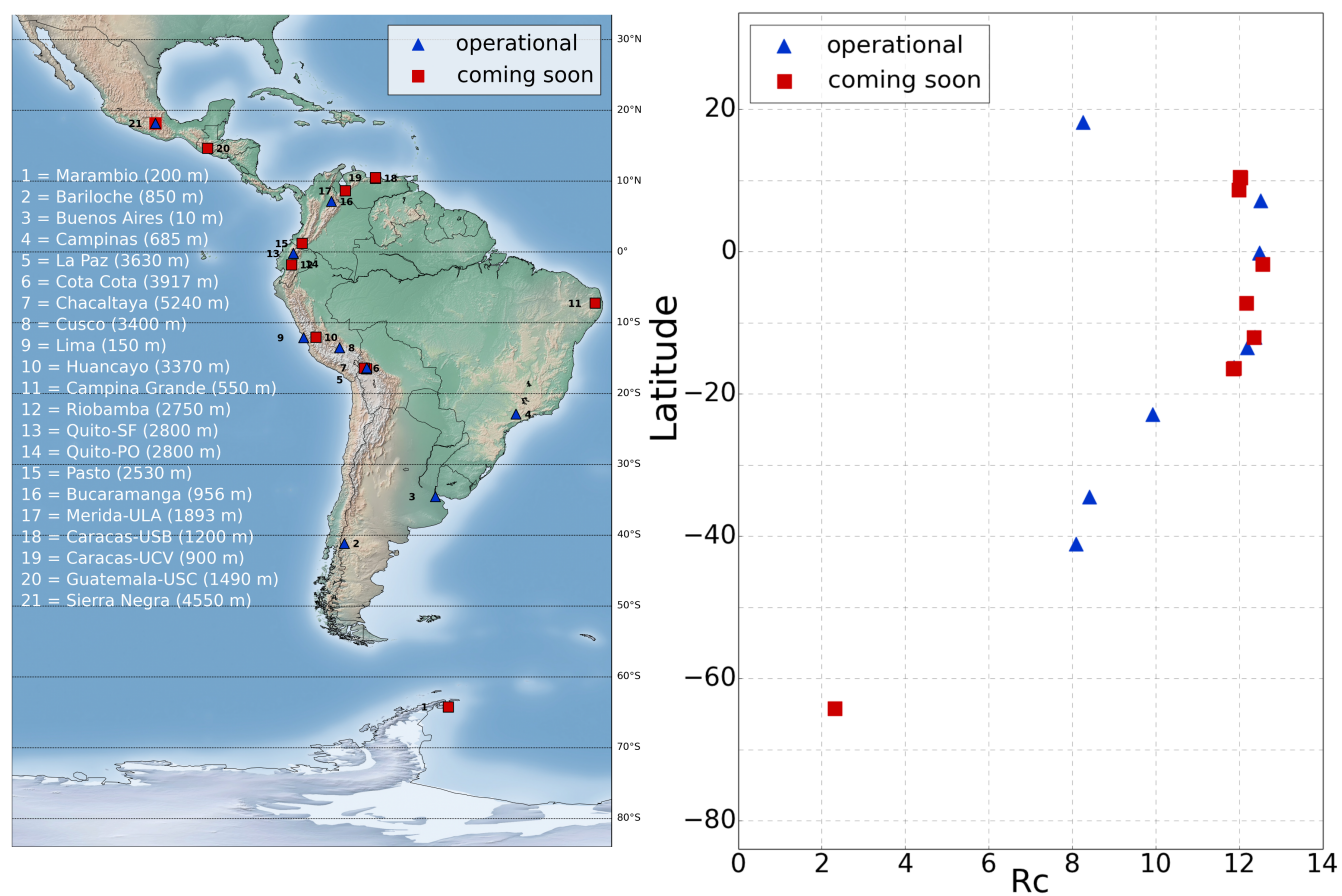

Figure 3: Left: map showing all LAGO sites in the Latin American region. The markers shows if the detector is operation, blue triangles, or if it is going to start measurements in the period 2015-2016, red squares. Right: rigidity cut off for the different LAGO sites, it can be seen in concordance with the map on the left. The different rigidities cut off shows the important of having a detection network like LAGO[7].

A compilation of the locations, number of detectors, altitudes, rigidity cut off, latitude and longitude, is made in table 1. It is worth mention some highlights of this table related to the LAGO science capabilities.

\section{Conclusion and final remarks}

In this paper we have shown a new concept developed by the LAGO Collaboration of an integral detector that includes the WCD working with an environmental station that has the capability of provides data for others communities.

It is also a highlight of the LAGO locations the very important difference in altitude sites across the Andes mountain range. This allow us to perform analyses of background radiation, space weather and low energy astroparticles.

The ultra long baseline "array" of WCD that goes from México to Antarctica bring different rigidity cut offs but with similar geographical longitudes 


\begin{tabular}{|c|c|ccccc|c|}
\hline \multirow{2}{*}{ Country } & \multirow{2}{*}{ Site } & Number of Altitude & \multicolumn{2}{|c|}{ Rigidity } & Latitude Longitude & \multirow{2}{*}{ Observations } \\
& & WCD & [m a.s.1.] cut off [GV] & [deg] & [deg] & \\
\hline \hline \multirow{5}{*}{ Argentina } & Bariloche & 3 & 850 & 8.10 & $41.15 \mathrm{~S}$ & $71.30 \mathrm{~W}$ & \\
& Buenos Aires & 1 & 10 & 8.41 & $34.54 \mathrm{~S}$ & $58.44 \mathrm{~W}$ & \\
& Marambio[16] & 1 & 200 & 2.31 & $64.24 \mathrm{~S}$ & $56.62 \mathrm{~W}$ & RC lowest \\
& & & & & & & value (south) \\
\hline \multirow{2}{*}{ Bolivia } & Chacaltaya & 3 & 5240 & 11.87 & $16.35 \mathrm{~S}$ & $68.13 \mathrm{~W}$ & Highest place \\
& Cota Cota & 1 & 3917 & 11.88 & $16.41 \mathrm{~S}$ & $68.5 \mathrm{~W}$ & \\
\hline \multirow{2}{*}{ Brazil } & Campinas & 1 & 685 & 9.93 & $22.9 \mathrm{~S}$ & $47.06 \mathrm{~W}$ & \\
& Campina Grande & 1 & 550 & 12.17 & $34.54 \mathrm{~S}$ & $58.44 \mathrm{~W}$ & Eastern place \\
\hline Colombia & Bucaramanga & 1 & 956 & 12.52 & $7.14 \mathrm{~S}$ & $73.12 \mathrm{~W}$ & \\
\hline \multirow{3}{*}{ Ecuador [17] } & Riobamba & 1 & 2750 & 12.55 & $1.81 \mathrm{~S}$ & $78.74 \mathrm{~W}$ & RC highest value \\
& Quito-SF & 1 & 2800 & 12.49 & $0.2 \mathrm{~S}$ & $78.5 \mathrm{~W}$ & \\
& Quito-PO & 1 & 2800 & 12.49 & $0.2 \mathrm{~S}$ & $78.5 \mathrm{~W}$ & \\
\hline Guatemala & Guatemala & 1 & 1490 & 10.32 & $14.63 \mathrm{~N}$ & $90.59 \mathrm{~W}$ & \\
\hline \multirow{2}{*}{ México [5] } & Sierra Negra & 4 & 4550 & 8.26 & $18.16 \mathrm{~N}$ & $97.95 \mathrm{~W}$ & RC lowest value \\
& & & & & & & (north) and western \\
\hline \multirow{3}{*}{ Perú [6] } & Lima & 2 & 150 & 12.37 & $12.10 \mathrm{~S}$ & $77.02 \mathrm{~W}$ & Large difference \\
& Cusco & 1 & 3400 & 12.19 & $13.52 \mathrm{~S}$ & $71.96 \mathrm{~W}$ & in altitude \\
& Huancayo & 1 & 3370 & 12.34 & $12.04 \mathrm{~S}$ & $75.30 \mathrm{~W}$ & at same RC \\
\hline \multirow{3}{*}{ Venezuela } & Caracas-UCV & 1 & 900 & 12.01 & $10.49 \mathrm{~N}$ & $66.89 \mathrm{~W}$ & \\
& Caracas-USB & 1 & 1200 & 12.02 & $10.41 \mathrm{~N}$ & $66.88 \mathrm{~W}$ & \\
& Mérida-ULA & 1 & 1893 & 11.99 & $8.63 \mathrm{~N}$ & $71.15 \mathrm{~W}$ & \\
\hline
\end{tabular}

Table 1: Table of the different locations of the LAGO sites.

The LAGO Project is being built as an extended astroparticle Observatory at a regional scale, operated by a collaborative and non-centralized network of institutions of nine Latin American countries and Spain.

\section{Acknowledgements}

The LAGO Collaboration is very thankful to the Pierre Auger Collaboration for its continuous support. Iván Sidelnik also wish to thank CONICET, CNEA and Instituto Balseiro.

\section{References}

[1] H. Asorey for the LAGO Collaboration, LAGO: the Latin American Giant Observatory, this proceedings (2015).

[2] D. Allard, I. Allekotte, C. Alvarez, H. Asorey, H. Barros, X. Bertou, O. Burgoa, M. Gomez Berisso, O. Martínez, P. Miranda Loza, T. Murrieta, G. Perez, H. Rivera, A. Rovero, O. Saavedra, H. Salazar, J. Tello, R. Ticona Peralda, A. Velarde, and L. Villaseñor, Use of water-Cherenkov detectors to detect Gamma Ray Bursts at the Large Aperture GRB Observatory (LAGO), Nuclear Instruments and 
Methods in Physics Research Section A: Accelerators, Spectrometers, Detectors and Associated Equipment 595 (Sept., 2008) 70-72.

[3] H. Asorey for the LAGO Collaboration, The LAGO Solar Project, Proc. 33th ICRC, Rio de Janerio, Brazil (2013).

[4] C. Sarmiento-Cano and others for the LAGO Collaboration, Analysis of Background Cosmic Ray Rate in the 2010-2012 Period from the LAGO Detectors at Chacaltaya, this proceedings (2015).

[5] A. Galindo and others for the LAGO Collaboration, Sensitivity of LAGO and Calibration of a Water-Cherenkov Detector in Sierra Negra, México., this proceedings (2015).

[6] L. Otiniano and F. Quispe. for the LAGO Collaboration, Development of a High Altitude LAGO Site in Peru, this proceedings (2015).

[7] M. Suarez and others for the LAGO Collaboration, The LAGO Space Weather Program: Directional Geomagnetic Effects, Background Fluence Calculations and Multi-Spectral Data Analysis, this proceedings (2015).

[8] http://www.raspberrypi.org/, Raspberry pi, June, 2015.

[9] http://http://www.arduino.cc/, Arduino, June, 2015.

[10] http://arduino.cc/en/Main/ArduinoShields, Arduino, June, 2015.

[11] http://www.atmel.com/dyn/resources/prod_documents/doc8161.pdf, Atmega328, June, 2015.

[12] http://www.raspberrypi.org/product/model-b plus/, Raspberry pi b model plus, June, 2015.

[13] C. L. Pryke and J. Lloyd-Evans, A high performance gps based autonomous event time-tagging system with application in a next generation extensive air shower array, Nuclear Instruments and Methods in Physics Research Section A: Accelerators, Spectrometers, Detectors and Associated Equipment 354 (1995), no. 2 560-566.

[14] A. J. Manucci et al., A global mapping techniques for GPS derived ionospheric total electron content measurement, Radio science 33(3) (1998) 565-582.

[15] J. J. Masías-Meza and S. Dasso, Geomagnetic effects on cosmic ray propagation under different conditions for buenos aires and marambio, argentina, arXiv preprint arXiv:1402.6274 (2014).

[16] S. Dasso and others for the LAGO Collaboration, A project to install water-cherenkov detectors in the antarctic peninsula as part of the LAGO detection network, this proceedings (2015).

[17] S. Vargas and others for the LAGO Collaboration, LAGO Ecuador, Implementing a Set of WCD Detectors for Space Weather Research: first results and further developments, this proceedings (2015). 